\title{
Modeling and Computer Simulation: Molecular Dynamics and Kinetic Monte Carlo
}

\author{
B.D. Wirth, M.J. Caturla, T. Diaz de la Rubia
}

\section{October 10, 2000}

U.S. Department of Energy

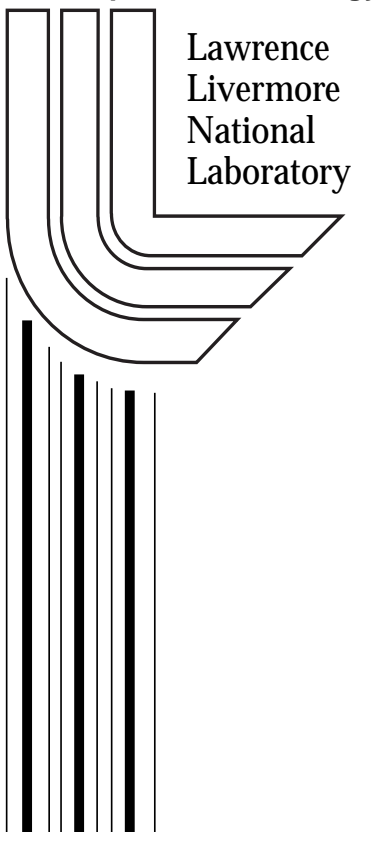




\section{DISCLAIMER}

This document was prepared as an account of work sponsored by an agency of the United States Government. Neither the United States Government nor the University of California nor any of their employees, makes any warranty, express or implied, or assumes any legal liability or responsibility for the accuracy, completeness, or usefulness of any information, apparatus, product, or process disclosed, or represents that its use would not infringe privately owned rights. Reference herein to any specific commercial product, process, or service by trade name, trademark, manufacturer, or otherwise, does not necessarily constitute or imply its endorsement, recommendation, or favoring by the United States Government or the University of California. The views and opinions of authors expressed herein do not necessarily state or reflect those of the United States Government or the University of California, and shall not be used for advertising or product endorsement purposes.

This work was performed under the auspices of the U. S. Department of Energy by the University of California, Lawrence Livermore National Laboratory under Contract No. W-7405-Eng-48.

This report has been reproduced directly from the best available copy.

Available to DOE and DOE contractors from the

Office of Scientific and Technical Information

P.O. Box 62, Oak Ridge, TN 37831

Prices available from (423) 576-8401

http://apollo.osti.gov/bridge/

Available to the public from the National Technical Information Service

U.S. Department of Commerce 5285 Port Royal Rd., Springfield, VA 22161

http://www.ntis.gov/

OR

Lawrence Livermore National Laboratory Technical Information Department's Digital Library http://www.llnl.gov/tid/Library.html 


\title{
Modeling and Computer Simulation: Molecular Dynamics and Kinetic Monte Carlo
}

\author{
B. D. Wirth, M. J. Caturla and T. Diaz de la Rubia \\ Chemistry and Materials Science Directorate \\ Lawrence Livermore National Laboratory \\ Livermore, CA USA
}

Recent years have witnessed tremendous advances in the realistic multiscale simulation of complex physical phenomena, such as irradiation and aging effects of materials, made possible by the enormous progress achieved in computational physics for calculating reliable, yet tractable interatomic potentials and the vast improvements in computational power and parallel computing. As a result, computational materials science is emerging as an important complement to theory and experiment to provide fundamental materials science insight. This article describes the atomistic modeling techniques of molecular dynamics (MD) and kinetic Monte Carlo (KMC), and an example of their application to radiation damage production and accumulation in metals. It is important to note at the outset that the primary objective of atomistic computer simulation should be obtaining physical insight into atomic-level processes.

Classical molecular dynamics is a powerful method for obtaining insight about the dynamics of physical processes that occur on relatively short time scales. Current computational capability allows treatment of atomic systems containing as many as $10^{9}$ atoms for times on the order of $100 \mathrm{~ns}\left(10^{-7} \mathrm{~s}\right)$. The main limitation of classical MD simulation is the relatively short times accessible. Kinetic Monte Carlo provides the ability to reach macroscopic times by modeling diffusional processes and time-scales rather than individual atomic vibrations. Coupling MD and KMC has developed into a powerful, multiscale tool for the simulation of radiation damage in metals.

\section{$\underline{\text { Molecular Dynamics Simulation }}$}

MD simulations of radiation damage were first performed by George Vineyard and coworkers in 1960 [1]. Since that time, numerous groups have utilized MD simulations to study the physics of primary defect production in high-energy displacement cascades [26] and atomic diffusion mechanisms [7-9] important to understanding radiation damage. A brief, introductory description of molecular dynamics simulation will be provided here. For additional information, the reader is referred to excellent tutorials by Allen and Tildesley [10] and Hoover [11]. 
MD computer simulation generates atomic trajectories for an N-particle system by direct numerical integration of Newton's equations of motion $(\vec{F}=m \vec{a})$ based on prescribed boundary and initial conditions. The atomic forces are derived from the potential energy function $\left(\vec{F}_{i}=-\partial U / \partial \vec{r}_{i}\right)$, which is, in general, a complicated function of the positions of all of the atoms in the system. Thus, the classical equations of atomic motion can be written as:

$$
\mathrm{m}_{\mathrm{i}} \frac{\mathrm{d}^{2} \mathrm{r}_{\mathrm{i}}}{\mathrm{dt}^{2}}=-\frac{\partial \mathrm{U}\left(\overrightarrow{\mathrm{r}}_{1}, \ldots, \overrightarrow{\mathrm{r}}_{\mathrm{N}}\right)}{\partial \overrightarrow{\mathrm{r}}_{\mathrm{i}}} \quad \mathrm{i}=1, \ldots, \mathrm{N}
$$

where $m_{i}$ is the mass, $\vec{r}_{i}$ is the position vector of atom $i$, and $U$ is the potential energy function. Starting from an initial configuration of the N-particle system and a specified potential energy function, the equations of motion (Eq. 1) are numerically integrated to provide the atomic trajectories $\left\{\overrightarrow{\mathrm{r}}_{\mathrm{i}}(\mathrm{t})\right\}=\left\{\overrightarrow{\mathrm{r}}_{\mathrm{i}}\left(\mathrm{t}_{\mathrm{o}}+\Delta \mathrm{t}\right)\right\}$ at any given time $\mathrm{t}$ by moving each particle forward in time by discrete steps $(\Delta \mathrm{t})$.

The basic steps in an MD simulation can be summarized as:

1) Specify the initial simulation model, e.g. the number of particles, the potential energy function, the initial system configuration, boundary conditions and the system state (desired pressure, temperature and volume).

2) Initialize the system by assigning each atom an initial kinetic energy, generally assigned from a Boltzmann distribution for the specified temperature.

3) Calculate the forces on each atom and integrate the equations of motion to determine each atoms acceleration, velocity and change in position.

4) Repeat step 3) until the desired time has elapsed.

\section{$\underline{\text { Monte Carlo }}$}

The Monte Carlo method was originally developed by von Neumann, Ulam and Metropolis to study the diffusion of neutrons in fissionable material on the Manhattan Project [12] and first applied to simulating radiation damage of metals by Doran [13] and later Heinisch and co-workers [14]. Monte Carlo utilizes random numbers to select from probability distributions and generate atomic configurations in a stochastic process [10], rather than the deterministic manner of MD simulations. While different Monte Carlo applications are used in computational materials science, we shall focus our attention on kinetic Monte Carlo (KMC) simulation of defect transport and damage accumulation as 
applied to the study of radiation damage. Additional information on Monte Carlo simulation techniques can be found in Allen and Tildesley [10].

KMC modeling of radiation damage involves tracking the location and fate of all defects, impurities and solutes as a function of time to predict microstructural evolution. The starting point in these simulations is the primary damage state, i.e. the spatially correlated locations of vacancy and interstitials produced in displacement cascades resulting from irradiation and obtained from MD simulations, along with the displacement or damage rate which sets the time scale for defect introduction. The appropriate activation energies for diffusion and dissociation, as well as the reactions that occur between species are also key input. In many cases, these activation energies can be obtained from MD simulations. The defects execute random diffusion jumps (in one, two or three dimensions depending on the nature of the defect) with a probability (rate) proportional to their diffusivity. Similarly, cluster dissociation rates are governed by a dissociation probability that is proportional to the binding energy of a particle to the cluster.

The basic steps in a KMC simulation can be summarized as:

1. Calculate the probability (rate) for a given event to occur.

2. Generate a random number to select an event from all possible events.

3. Increment the simulation time based on the inverse sum of the rates of all possible events $\left(\Delta t=\frac{\chi}{\sum_{i} N_{i} R_{i}}\right)$, where $\chi$ is a random deviate that assures a Poisson distribution in time steps, $\mathrm{N}$ and $\mathrm{R}$ are the number and rate of each event $\mathrm{i}$.

4. Perform the selected event and all spontaneous events as a result of the event performed.

5. Repeat steps 1 through 4 until the desired simulation condition is reached.

\section{$\underline{\text { Radiation Damage in } \mathrm{Cu}}$}

Irradiation of copper by high-energy neutrons and ions produces significant changes to material microstructure and its mechanical properties. Post-irradiation microstructural characterization of $\mathrm{Cu}$ has been extensively used to quantify the number and types of defects formed as a function of radiation exposure conditions [15-17]. Below doses of 0.1 displacements per atom (dpa), most of the defects observed in transmission electron microscopy (TEM) examination are stacking fault tetrahedra (SFT), which have a constant mean size of about $2.5 \mathrm{~nm}$ over a wide range of irradiation dose and temperature [15-17]. 
To provide insight into the physical processes responsible for microstructural evolution in irradiated $\mathrm{Cu}$, we have performed a linked MD and KMC simulation [18]. Displacement cascades result from the collision of high-energy particles (e.g., neutron, alpha, proton or heavy ion) with lattice atoms and, in most metals, evolve over a few tens of picoseconds to produce a spatially correlated distribution of vacancies, self-interstitials and their clusters. MD simulation is an ideal technique for studying the evolution of displacement cascades, as a result of the short temporal evolution. Figure 1 shows an example of the displacement cascade evolution produced in copper by a $20 \mathrm{keV}$ displacement cascade. Large vacancy and self-interstitial clusters are produced within a few tens of picoseconds as the kinetic energy is dissipated and the cascade cools. The large vacancy clusters collapse to form stacking fault tetrahedra (SFT) within a few picoseconds at room temperature, as observed in MD simulations [19]. The formation of large point defect clusters in high-energy displacement cascades is one example of the improved understanding of radiation damage that has resulted from atomistic simulations.

An MD database of the number and spatial distribution of defects produced in displacement cascades provides key input into a KMC simulation of damage accumulation, along with information about the character and energetics of the vacancy, self-interstitial and their clusters. Figure 2 shows a comparison between a number of experimental results $[16,17]$ and KMC predictions of the visible defect accumulation in $\mathrm{Cu}$, when neutron irradiated near room temperature. Both the visible defect density and the KMC prediction that over $90 \%$ of the visible point defect clusters are vacancy SFT in irradiated $\mathrm{Cu}$ are in excellent agreement with experiments [15-17]. Further, the KMC results predict a constant mean SFT size with increasing irradiation, once again in good agreement with experimental observations [15-17].

\section{REFERENCES:}

1. J. B. Gibson, A. N. Goland, M. Milgram, and G. H. Vineyard, Phys. Rev. 120 (1960) 1229

2. $\quad$ A. F. Calder and D. J. Bacon, J. Nuc. Mat. 207 (1993) 25.

3. H. L. Heinisch and B. N. Singh, Phil Mag A 67 (1993) 407.

4. M. T. Robinson, J. Nuc. Mat., 216 (1994) 1.

5. W. J. Phythian, R. E. Stoller, A. J. E. Foreman, A. F. Calder, and D. J. Bacon, J. Nuc. Mat. 223 (1995) 245.

6. $\quad$ R. S. Averback and T. Diaz de la Rubia, Solid State Physics, 51 (1998) 281. 
7. Y. N. Osetsky, M. Victoria, A. Serra, S. I. Golubov, and V. Priego, J. Nucl. Mat., 251 (1997) 34.

8. N. Soneda, and T. Diaz de la Rubia, Phil Mag A 78 (1998) 995.

9. B. D. Wirth, G. R. Odette, D. Maroudas and G. E. Lucas, J. Nuc. Mat. 244 (1997) 185.

10. M. P. Allen and D. J. Tildesley, "Computer Simulation of Liquids", Clarendond Press, Oxford (1990).

11. W. G. Hoover, "Computational Statistical Mechanics", Elsevier Science Publishers, Amsterdam (1991).

12. $\quad$ N. Metropolis and S. Ulam, J. Am. stat. Ass. 44 (1949) 335.

13. D. G. Doran, Irradiation Effects 2 (1970) 249.

14. H. L. Heinisch and B. N. Singh, J. Nuc. Mat. 251 (1997) 77.

15. S. J. Zinkle, J. Nuc. Mat., 150 (1987)140.

16. B. N. Singh and S. J. Zinkle, J. Nuc. Mat., 206 (1993) 212.

17. Y. Dai, M. Victoria, Mat. Res. Soc. Symp. Proc., 439 (1997) 319.

18. M. J. Caturla, N. Soneda, E. A. Alonso, B. D. Wirth and T. Diaz de la Rubia, J. Nuc. Mat., 276 (2000) 13.

19. B. D. Wirth, V. Bulatov and T. Diaz de la Rubia, J. Nuc. Mat. (2000) in press. 

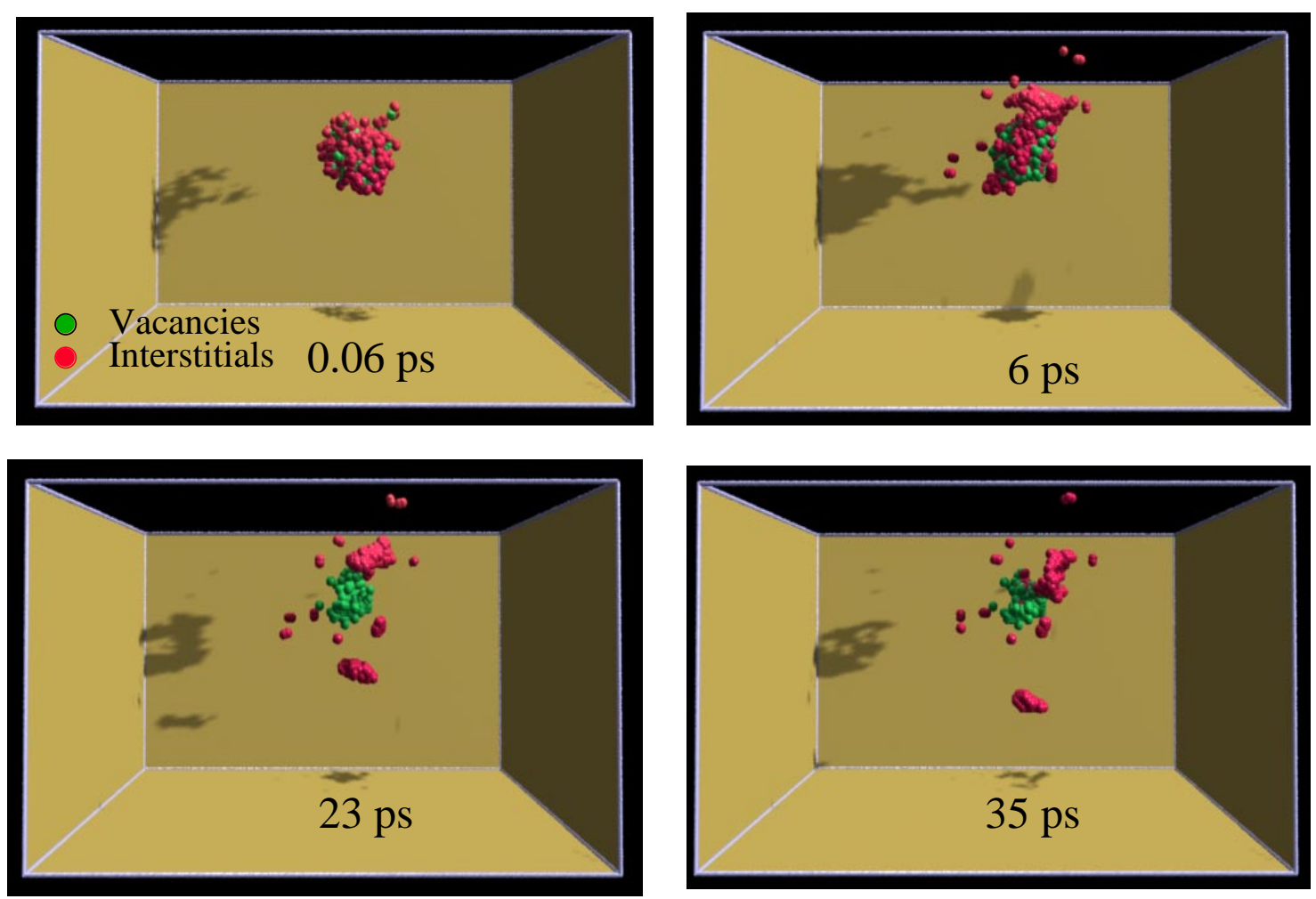

Figure 1 - Cascade evolution for a $20 \mathrm{keV} \mathrm{Cu}$ recoil in $\mathrm{Cu}$. Red and green dots indicate the position of self-interstitial atoms and vacancies, respectively. Observe the migration of a large self-interstitial cluster between 23ps and 35 ps. 


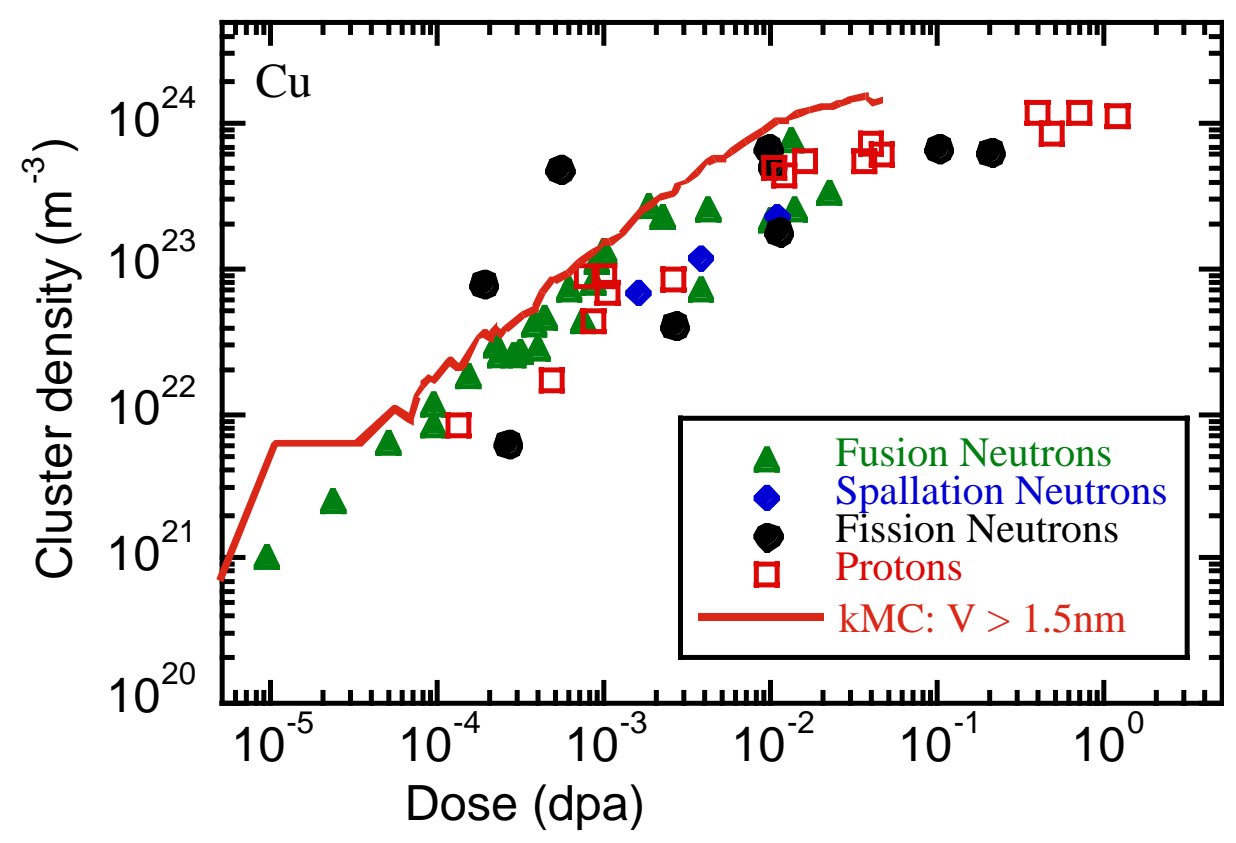

Figure 2 - Visible cluster density with increasing irradiation dose in irradiated $\mathrm{Cu}$. KMC predictions (solid line) [18] are in good agreement with experimental data for irradiation by fission, fusion and spallation neutrons and high energy protons [15-17]. 